\title{
Patterned arrays of vertically aligned carbon nanotube microelectrodes on carbon films prepared by thermal chemical vapor deposition
}

\author{
Xianming Liu, ${ }^{\dagger}$ Keith H. R. Baronian, ${ }^{\star}$ and Alison J. Downard ${ }^{* \dagger}$
}

MacDiarmid Institute for Advanced Materials and Nanotechnology, Department of

Chemistry, University of Canterbury, Private Bag 4800, Christchurch 8140, New

Zealand, and Christchurch Polytechnic Institute of Technology, PO Box 540,

Christchurch 8140, New Zealand

*To whom correspondence should be addressed.

email: Alison.downard@canterbury.ac.nz; phone: +64-3-364501; fax: +64-336421100

${ }^{\dagger}$ University of Canterbury

* Christchurch Polytechnic Institute of Technology

\begin{abstract}
A straightforward procedure is described for preparation of arrays of microdisk electrodes comprising bundles of vertically aligned carbon nanotubes (VACNTs). The arrays are fabricated by thermal chemical vapor deposition synthesis directly on a planar carbon film support. Use of standard micro- and nanolithography procedures for patterning the bilayer catalyst spots enables arrays to be grown with controlled electrode diameters and spacings. The minimum accessible VACNT
\end{abstract}


bundle diameter, and hence microelectrode diameter, is $2 \mu \mathrm{m}$. After insulating the arrays with SU-8 epoxy and exposing the VACNT ends by polishing or treating with $\mathrm{O}_{2}$ plasma, the microdisk electrodes exhibit attractive electrochemical properties.

\begin{tabular}{llll}
\hline \multicolumn{2}{c}{ Table 1. Layout of Catalyst Microdisk Patterns and Patterning Method } \\
\cline { 2 - 3 } & & & minimum spacing \\
disk diameter $(\mu \mathrm{m})$ & patterning method & number of disks & between disks $(\mu \mathrm{m})$ \\
100 & & 16 & 900 \\
10 & shadow mask & 77 & 155 \\
2 & EBL & 400 & 50 \\
\hline
\end{tabular}

Table 2. Voltammetric data (scan rate $\left.=100 \mathrm{mV} \mathrm{s}^{-1}\right)$ for $1 \mathrm{mM}$ FcOH in $0.1 \mathrm{M} \mathrm{KCl}$

\begin{tabular}{lllll} 
array & treatment & $i_{\text {lim }}(\mathrm{nA})$ & $i_{\text {cal }}(\mathrm{nA})^{a}$ & $E_{3 / 4}-E_{1 / 4}(\mathrm{mV})$ \\
$16 \times 100 \mu \mathrm{m}$ & polished & $190^{b}$ & 216 & 61 \\
$77 \times 10 \mu \mathrm{m}$ & polished & 141 & 104 & 63 \\
$77 \times 10 \mu \mathrm{m}$ & $\mathrm{O}_{2}$ plasma & 178 & 104 & 67 \\
$400 \times 2 \mu \mathrm{m}$ & polished & 157 & 108 & 72 \\
$400 \times 2 \mu \mathrm{m}$ & $\mathrm{O}_{2}$ plasma & 152 & 108 & 70 \\
${ }^{a}$ Calculated from eqn (1) assuming $D=7.0 \times 10^{-6} \mathrm{~cm}^{2} \mathrm{~s}^{-1}$ \\
${ }^{b}$ voltammogram recorded with scan rate $=5 \mathrm{mV} \mathrm{s}^{-1}$ \\
\hline
\end{tabular}

\section{Figure captions}

Figure 1. SEM images of patterned VACNT pillars grown using thermal CVD: (a) array of $16 \times 100 \mu \mathrm{m}$ pillars; (b) single $100 \mu \mathrm{m}$ pillar; (c) array of 77 x $10 \mu \mathrm{m}$ pillars; (d) single $10 \mu \mathrm{m}$ pillar; (e) array of $400 \times 2 \mu \mathrm{m}$ pillars; (f) single $2 \mu \mathrm{m}$ pillar

Figure 2. SEM images of VACNT microdisk electrode arrays, insulated with SU-8 epoxy. (a) $77 \times 10 \mu \mathrm{m}$ array and (b) single $10 \mu \mathrm{m}$ electrode, after polishing; (c) $77 \mathrm{x}$ 
$10 \mu \mathrm{m}$ array and (d) single $10 \mu \mathrm{m}$ electrode, after treatment with $\mathrm{O}_{2}$ plasma; (e) $400 \mathrm{x}$ $2 \mu \mathrm{m}$ array and (f) single $2 \mu \mathrm{m}$ electrode after polishing; (g) $400 \times 2 \mu \mathrm{m}$ array and (h) single $2 \mu \mathrm{m}$ electrode, after treatment with $\mathrm{O}_{2}$ plasma.

Figure 3. Steady-state voltammograms of $1 \mathrm{mM} \mathrm{FcOH}$ in $0.1 \mathrm{M} \mathrm{KCl}$ obtained at a) polished $16 \times 100 \mu \mathrm{m}$ array; b) polished $77 \times 10 \mu \mathrm{m}$ array; c) $\mathrm{O}_{2}$ plasma-treated $77 \times$ $10 \mu \mathrm{m}$ array; d) polished $400 \times 2 \mu \mathrm{m}$ array and e) $\mathrm{O}_{2}$ plasma-treated $400 \times 2 \mu \mathrm{m}$ array. a) scan rate $=5 \mathrm{mV} \mathrm{s}^{-1} ;$ b) - e) scan rate $=100 \mathrm{mV} \mathrm{s}^{-1}$. All scale bars represent $20 \mathrm{nA}$. 


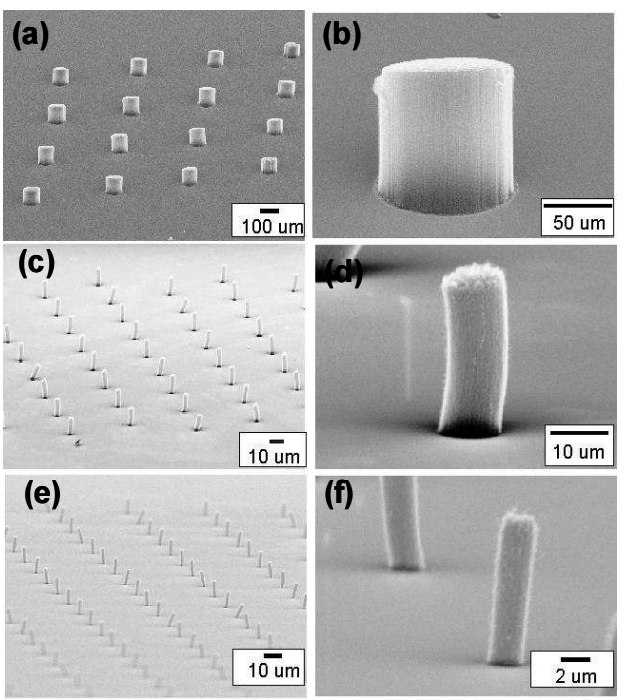

Figure 1. 


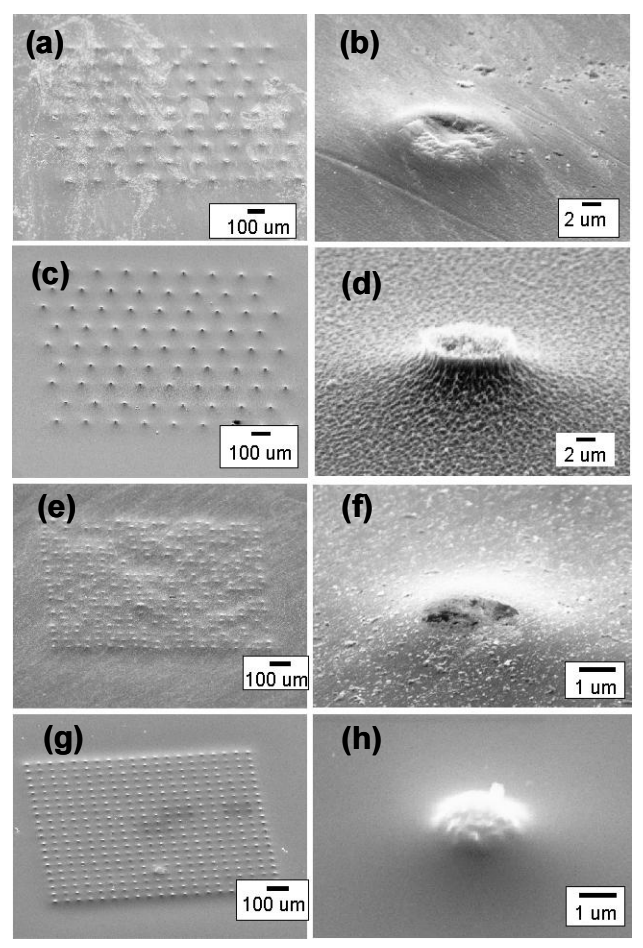

Figure 2 
(a)

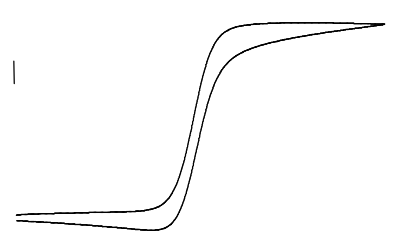

(b)

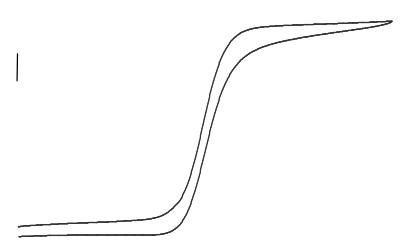

(c)

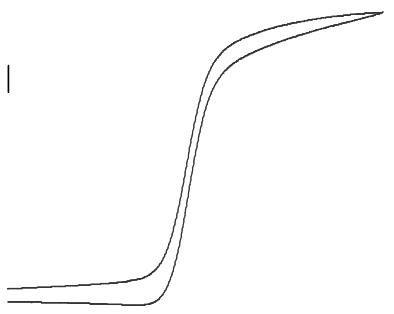

(d)

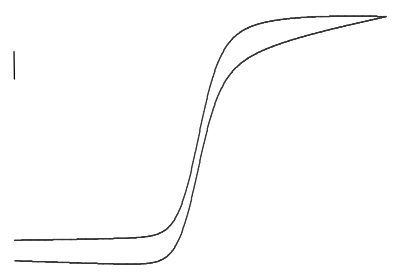

(e)

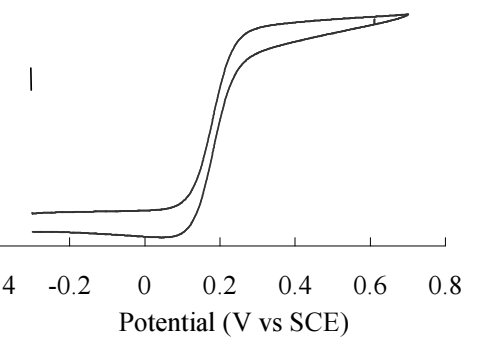

Figure 3 